\title{
Multicenter evaluation of breast cancer patients' satisfaction and experience with oncology telemedicine visits during the COVID-19 pandemic
}

\author{
Alexandra Bizot ${ }^{1}$, Maryam Karimi ${ }^{2,3}$, Elie Rassy ${ }^{10}{ }^{1}$, Pierre Etienne Heudel ${ }^{4}$, Christelle Levy ${ }^{5}$, Laurence Vanlemmens ${ }^{6}$, Catherine Uzan $^{7}$, \\ Elise Deluche ${ }^{8}$, Dominique Genet ${ }^{9}$, Mahasti Saghatchian ${ }^{10}$, Sylvie Giacchetti ${ }^{11}$, Juline Grenier ${ }^{12}$, Anne Patsouris ${ }^{13}$, Véronique Dieras ${ }^{14}$, \\ Jean-Yves Pierga $\mathbb{D D}^{15}$, Thierry Petit ${ }^{16}$, Sylvain Ladoire ${ }^{17}$, William Jacot ${ }^{10}{ }^{18}$, Marc-Antoine Benderra ${ }^{19}$, Anne De Jesus ${ }^{20}$, \\ Suzette Delaloge (iD) ${ }^{1}$, Matteo Lambertini $\mathbb{D}^{21,22}$ and Barbara Pistilli $\mathbb{D}^{1 \times}$
}

(C) The Author(s), under exclusive licence to Springer Nature Limited 2021

INTRODUCTION: During the COVID-19 pandemic, teleconsultation was implemented in clinical practice to limit patient exposure to COVID-19 while monitoring their treatment and follow-up. We sought to examine the satisfaction of patients with breast cancer (BC) who underwent teleconsultations during this period.

METHODS: Eighteen centres in France and Italy invited patients with $\mathrm{BC}$ who had at least one teleconsultation during the first wave of the COVID-19 pandemic to participate in a web-based survey that evaluated their satisfaction (EORTC OUT-PATSAT 35 and Telemedicine Satisfaction Questionnaire [TSQ] scores) with teleconsultation.

RESULTS: Among the 1299 participants eligible for this analysis, 53\% of participants were undergoing standard post-treatment follow-up while 22 and $17 \%$ were currently receiving active anticancer therapy for metastatic and localised cancers, respectively. The mean satisfaction scores were 77.4 and 73.3 for the EORTC OUT-PATSAT 35 and TSQ scores, respectively. In all, $52.6 \%$ of participants had low/no anxiety. Multivariable analysis showed that the EORTC OUT-PATSAT 35 score correlated to age, anxiety score and teleconsultation modality. The TSQ score correlated to disease status and anxiety score.

CONCLUSION: Patients with BC were satisfied with oncology teleconsultations during the COVID-19 pandemic. Teleconsultation may be an acceptable alternative follow-up modality in specific circumstances.

British Journal of Cancer (2021) 125:1486-1493; https://doi.org/10.1038/s41416-021-01555-y

\section{INTRODUCTION}

Eighteen months after the coronavirus disease 2019 (COVID-19) pandemic began in China, the number of active cases that reached 145 million cases has placed significant strain on the medical community globally [1]. The rapidly growing case numbers have overwhelmed the health delivery systems and imposed unprecedented challenges to maintain essential healthcare services [2]. Unsurprisingly, cancer care was disrupted by the decreases and delays in detecting new cancers and delivering appropriate treatment [3]. Moreover, patients with cancer seem to be particularly vulnerable to severe complications of COVID-19 because of their immunosuppressive state induced by the malignancy and anticancer therapy [4,5]. Multiple oncology societies have published consensus guidelines to amend cancer care and mitigate the negative effects of the COVID-19 pandemic [6-8]. Healthcare providers accommodated adjustments to compensate for these changes by minimising the number of hospital visits and preventing anticancer treatment-induced complications of COVID-19 [9, 10].

Many centres have adopted teleconsultation in daily practice to limit patient exposure to COVID-19 while monitoring their

\footnotetext{
${ }^{1}$ Medical Oncology Department, Gustave Roussy, Villejui, France. ${ }^{2}$ Department of Biostatistics and Epidemiology, Gustave Roussy, University Paris-Saclay, Villejuif, France.

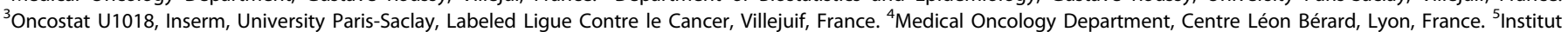

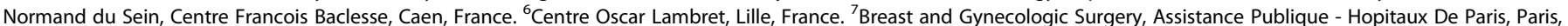

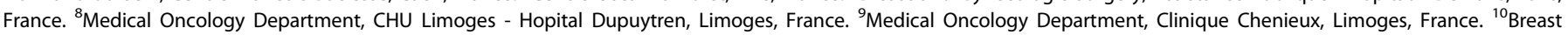

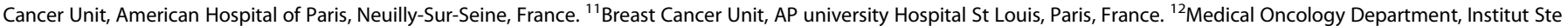

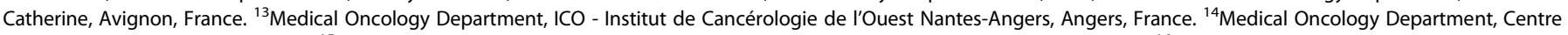

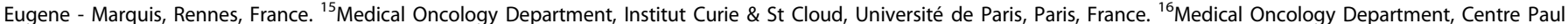

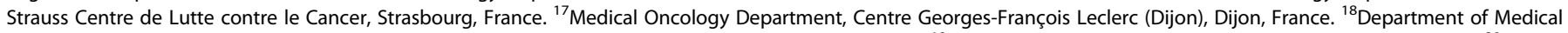

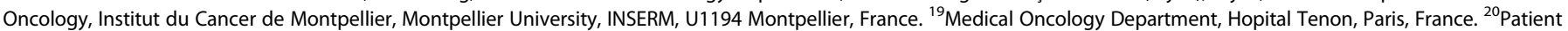

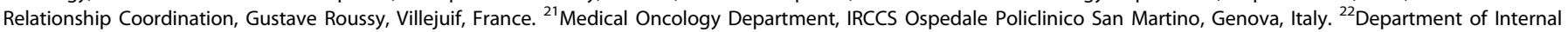
Medicine and Medical Specialties (DiMI), School of Medicine, University of Genova, Genova, Italy. ${ }^{凶}$ email: barbara.pistilli@gustaveroussy.fr
} 
treatment and follow-up [11]. Teleconsultation is already implemented in USA and Northern European countries to ensure healthcare services across long distances, through the use of telecommunications and information technologies. During the past year, an expert consensus has favoured teleconsultation mainly among patients undergoing intravenous chemotherapies for early-stage breast cancer, receiving systemic treatments for metastatic breast cancer or during follow-up for breast cancer [12-14]. Given the knowledge gap in evaluating the preference of patients with this approach, we sought to examine the satisfaction of patients with breast cancer who underwent teleconsultations during the COVID-19 pandemic.

\section{PARTICIPANTS AND METHODS Study design}

This prospective, observational, multicentric survey enrolled patients from April 6 to May 25, 2020 from 17 hospitals and cancer centres in France and 1 hospital in Italy. All patients with breast cancer, who were followed through at least one telehealth/at-phone visit during the COVID-19 pandemic, received by email the link to fill a one-time satisfaction questionnaire along with an information letter between 2 and 14 days after the teleconsultation. Each questionnaire was filled only once by each participant, according to the used Internet Protocol address. Neither reminders nor second invitations were sent to any of the participants. Therefore, patients who had multiple teleconsultations answered the questionnaire only once.

The online questionnaire was completely anonymous. An information letter notified the participants that completing the questionnaire infers an inherent agreement to their participation in the study. The study was examined and approved by the internal review board at Gustave Roussy, Villejuif, France on April 6, 2020.

\section{Questionnaire}

The questionnaire was based on pre-existing validated scales and was designed into four sections after a comprehensive literature review. The first section enclosed seven items to collect information about the participants (age, family situation, accommodation type and region), the disease status (localised cancer, metastatic cancer, standard follow-up or other), teleconsultation situation (alone, as a couple, with family and other) and modality of teleconsultation (video or phone). The option "other" was included in some questions to allow participants to provide responses that were not defined in the listed answers.

The second section included 11 items derived from the EORTC OUTPATSAT 35 and focused on the physicians' technical skills (knowledge, experience, assessment of physical symptoms), interpersonal skills (interest, willingness to listen), provision of information (about the disease, medical tests and treatment) and availability [15]. The corresponding French and Italian validated versions of the EORTC OUT-PATSAT 35 were used for this study. The items that were removed from the original EORTC OUT-PATSAT 35 questionnaire were those relevant to the nurse evaluation section and the physical environment organisation. The response format consisted of a five-point Likert scale: 1-poor, 2-fair, 3-good, 4-very good, and 5-excellent.

The third section aimed to evaluate the satisfaction of the participants with the teleconsultation experience according to the 15-question Telemedicine Satisfaction Questionnaire (TSQ), which was initially validated in diabetic patients and recently in oncology patients $[16,17]$. The TSQ was translated into French and Italian using the translation/back-translation method, a process through which the questionnaire is translated from English to French and Italian, and then it is translated back to English by a different translator who is unaware of the questionnaire's initial wording in English. The English version was compared by the investigators for final approval. The third question of the TSQ was redundant in the EORTC OUTPATSAT 35 and was consequently removed from the last version of the questionnaire. The response format consisted of a five-point Likert scale: 1 - poor, 2-fair, 3-good, 4-very good, and 5-excellent.

The fourth section used the Hospital Anxiety Depression Scale subscale for anxiety (HADS-A) to assess the patients' anxiety and psychological distress associated with the global healthcare crisis $[18,19]$. HADS-A is a validated 14-item scale, composed of 7 items assessing the intensity of anxiety and 7 items evaluating the intensity of depressive symptoms. For this study, only the 7 questions exploring patients' anxiety were retained in the questionnaire. Each question was scored from 0 to 3, with a higher score reflecting a higher level of anxiety.

Last, two items were added to reflect on the participants' point of view on teleconsultation and its potential use in the future.

\section{Statistical analysis}

Descriptive statistics were used to describe the participants' sociodemographic and clinical characteristics, with frequency and proportion for qualitative variables and mean and standard deviation (SD) for quantitative variables. The corresponding regions of each participant were categorised into COVID-19 hotspots and non-hotspots at the time of the survey (Regions Auvergne-Rhône-Alpes, Bourgogne-Franche-Comté, Grand-Est, Hauts-de-France, Ile de France from France and Liguria from Italy were considered as hotspots). The corresponding Likert score of the EORTC OUT-PATSAT 35 and TSQ items was linearly transformed to a 0-100 scale, with a higher score reflecting a higher level of satisfaction. The anxiety level (varying between 0 and 21 for each patient) was discretized into no/low anxiety, possible/minimal anxiety, anxiety and severe anxiety according to the HADS-A cutoffs at 7, 10 and 14, respectively. Spearman correlation was used to examine the correlation of the anxiety score (HADS-A) and satisfaction scales (EORTC OUT-PATSAT 35 and TSQ). We first investigated the values of satisfaction scales (EORTC OUT-PATSAT 35 and TSQ) across different characteristics (anxiety class, age class, disease status, accommodation type, family situation, teleconsultation situation and teleconsultation modality and region). Specifically, the mean value of each score (EORTC OUT-PATSAT 35 and TSQ) was calculated by performing univariable linear regression models with the score as the response variable and each characteristic as the explanatory variable. In order to evaluate the effect of each characteristic on the changes in the mean score (EORTC OUT-PATSAT 35 and TSQ), we performed multivariable linear regression models with the score as the response variable and all characteristics as explanatory variables. The linear regression coefficients obtained from these multivariable analyses represented the average adjusted contribution of each covariate to the score. Further, participants were categorised into two subsets according to the EORTC OUT-PATSAT 35 and TSQ scores; those scoring within the first quartile were assigned to the "poor satisfaction" group. A multivariable logistic regression model was used to investigate characteristics that were associated with a poor satisfaction score. Results were reported by odds ratios (ORs) and $95 \%$ confidence intervals (Cls). We considered a nominal significance level of 0.05. All statistical analyses were conducted using R V.3.6.2 in the RStudio environment.

\section{RESULTS}

\section{Patient socio-demographic and clinical characteristics}

In total, 3722 patients were approached for inclusion and 2288 did not reply to the study invitation for unknown reasons. Of the 1434 participants (38.5\%) who answered the questionnaire, 135 participants were excluded from the analysis for the following reasons: 100 participants did not complete all the items of the survey and hence 1 of the 3 score was missing, 35 participants did not report the age range, and 7 participants fell into the 2 categories. In total, 1299 participants were considered for the purpose of this study. The majority of participants were aged $\geq 50$ years $(n=1042 ; 72.6 \%)$ and lived with their spouse or family members $(n=1100 ; 76.7 \%)$. Approximately half of the participants ( $n=764 ; 53.3 \%$ ) were undergoing standard post-treatment followup (with or without ongoing endocrine therapy) and the remaining were receiving anticancer therapy for metastatic and localised cancers (22.2 and $16.8 \%$, respectively). The teleconsultations were predominantly by phone $(n=1294 ; 90.2 \%)$ and performed without the presence of a companion $(n=948 ; 68.6 \%)$ (Table 1). The clinical and socio-demographic characteristics of patients who used phone- and video-based teleconsultation are reported in Supplementary Table 1.

\section{Perceived satisfaction with telehealth visits}

In the overall population, the mean scores were $77.4(S D=17.0)$, $73.3(S D=15.5)$ and $7.2(S D=4.1)$ for the EORTC OUT-PATSAT 35 score, TSQ score and HADS-A, respectively. Notably, almost half 
Table 1. Participants' socio-demographic, clinical characteristics and scores.

\begin{tabular}{|c|c|c|c|c|}
\hline & & $\begin{array}{l}\text { Overall }(N=1434) \\
n(\%)\end{array}$ & $\begin{array}{l}\text { France }(N=1338) \\
n(\%)\end{array}$ & $\begin{array}{l}\text { Italy }(N=96) \text {, } \\
n(\%)\end{array}$ \\
\hline \multirow{4}{*}{ Age in years } & $40-49$ & $265(18.5)$ & $241(18.0)$ & $24(25.0)$ \\
\hline & $60-69$ & $367(25.6)$ & $351(26.2)$ & $16(16.7)$ \\
\hline & $\geq 70$ & 257 (17.9) & $255(19.1)$ & $2(2.1)$ \\
\hline & (Missing) & $35(2.4)$ & $33(2.5)$ & $2(2.1)$ \\
\hline Accommodation type & Other & $10(0.7)$ & $10(0.7)$ & $0(0.0)$ \\
\hline \multirow[t]{2}{*}{ Region } & Hotspots & $989(69.0)$ & $896(67.0)$ & $93(96.9)$ \\
\hline & Non-hotspots & $445(31.0)$ & $442(33.0)$ & $3(3.1)$ \\
\hline \multirow[t]{2}{*}{ Family situation } & Alone & $258(18.0)$ & $249(18.6)$ & $9(9.4)$ \\
\hline & As a couple & $568(39.6)$ & $551(41.2)$ & $17(17.7)$ \\
\hline \multirow{3}{*}{ Disease status } & Metastatic cancer & $318(22.2)$ & $316(23.6)$ & $2(2.1)$ \\
\hline & Standard follow-up & $764(53.3)$ & $701(52.4)$ & $63(65.6)$ \\
\hline & Other & $111(7.7)$ & $104(7.8)$ & $7(7.3)$ \\
\hline \multirow[t]{3}{*}{ Teleconsultation setting } & Alone & $984(68.6)$ & $922(68.9)$ & $62(64.6)$ \\
\hline & Family & 429 (29.9) & 398 (29.7) & $31(32.3)$ \\
\hline & Other & $21(1.5)$ & $18(1.3)$ & $3(3.1)$ \\
\hline \multirow[t]{2}{*}{ Teleconsultation type } & Video & $140(9.8)$ & $140(10.5)$ & $0(0.0)$ \\
\hline & Phone & $1294(90.2)$ & $1198(89.5)$ & $96(100.0)$ \\
\hline \multirow[t]{2}{*}{ EORTC OUT-PATSAT 35 score $(0-100)$} & Mean (SD) & $77.4(17.0)$ & $78.1(16.7)$ & $67.6(18.2)$ \\
\hline & (Missing) & 88 & 83 & 5 \\
\hline TSQ score $(0-100)$ & Mean (SD) & $73.3(15.5)$ & $73.4(15.4)$ & $72.3(16.5)$ \\
\hline $\begin{array}{l}\text { Hospital Anxiety Depression Scale subscale for } \\
\text { anxiety (4 levels) }\end{array}$ & (Missing) & $106(7.4)$ & $101(7.5)$ & $5(5.2)$ \\
\hline
\end{tabular}

HADS Hospital Anxiety Depression Scale subscale for anxiety, $n$ number of patients, $S D$ standard deviation.

of the population experienced no/low anxiety $(52.6 \%$ had HADS$A \leq 7$ ) and only $5.2 \%$ of the population had severe anxiety (HADS$A \geq 15$ ) (Table 1).

EORTC OUT-PATSAT 35 score. Univariable linear regression models showed significant differences in the mean EORTC OUTPATSAT 35 score across anxiety levels with a lower mean among patients with severe anxiety compared to those with no/low anxiety $(p<0.01)$. The negative Spearman correlation coefficient between HADS-A as a continuous variable and EORTC OUTPATSAT 35 score confirmed this finding (Supplementary Fig. 1). The results also showed differences in mean EORTC OUT-PATSAT 35 score across age groups (lower mean among groups 40-49 and 50-59 compared to younger patients) and teleconsultation modality (higher satisfaction level for those with video consultation compared to phone consultation) (Fig. 1). Multivariable analysis confirmed the significant association between EORTC OUT-PATSAT 35 score, anxiety level and teleconsultation modality. Differences in mean EORTC POUT-PATSAT 35 was found between "Possible anxiety" and "Severe anxiety" groups compared to "No/ low anxiety" patients $(\beta=-3.8, p=0.001$ and $\beta=-5.9, p=0.005$, respectively). Lower mean satisfaction score was observed for those patients who performed the teleconsultation by phone compared to video consultation $(\beta=-8.2, p<0.001)$ (Table 2). Poor satisfaction was associated with severe anxiety compared to no/low anxiety, the odds of not being satisfied (in terms of EORTC OUT-PATSAT 35) increased by a factor of 2.1 compared to those with no/low anxiety $(\mathrm{OR}=2.1,95 \% \mathrm{Cl} 1.2-3.6)$. Being in the $40-49$ or 50-59 age groups increased the odds of poor satisfaction by a factor of 2.2 and 2.3 compared to younger patients (<40 years) $(\mathrm{OR}=2.2[95 \% \mathrm{Cl} 1.1-4.3]$ and $2.3[95 \% \mathrm{Cl} 1.2-4.4])$. The results showed a significant association with the teleconsultation modality, as phone teleconsultations increased the odds of poor satisfaction by a factor of 2.7 compared to video consultations (OR $=2.7,95 \% \mathrm{Cl} 1.5-4.9$ ) (Table 3).

TSQ score. Univariable regressions showed a lower mean TSQ score among patients with "Possible anxiety", "Anxiety" and "Severe anxiety" compared to those with no/low anxiety $(p<0.05)$. The results showed a higher mean TSQ score for those with video 


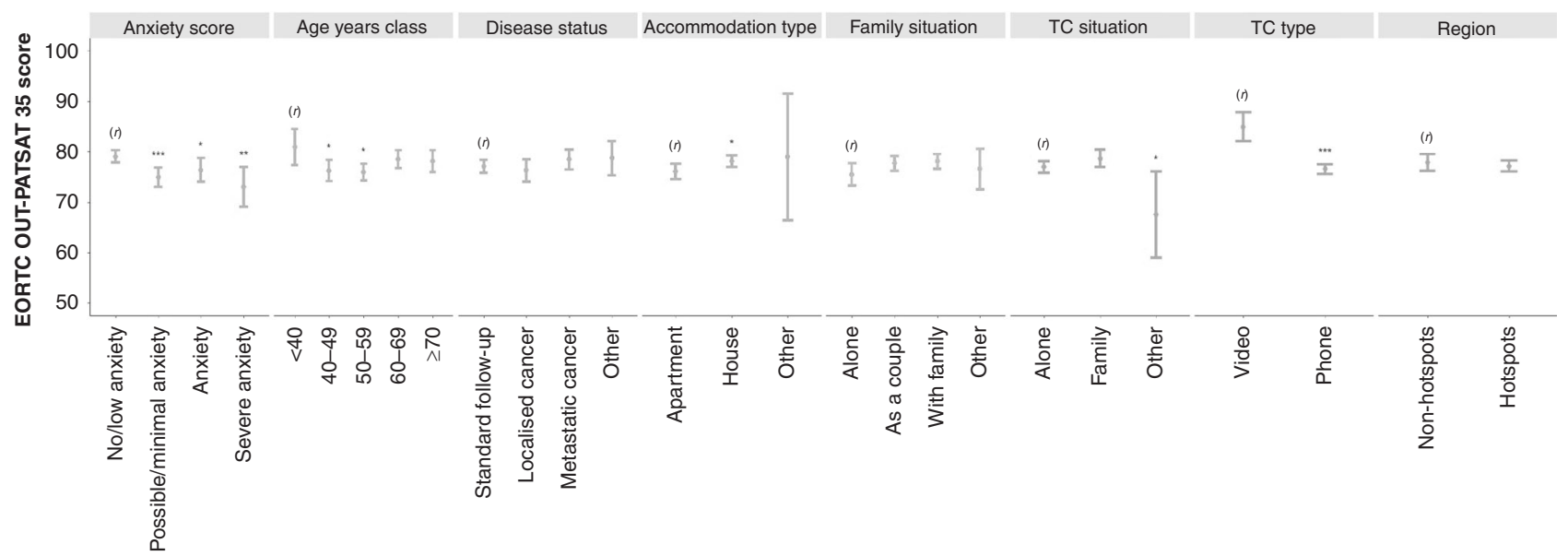

Fig. 1 Forest plot of the univariate analysis for the EORTC OUT-PATSAT 35 score distribution by different characteristic factors. For each factors' category, the point estimate of the mean score is represented by a bullet, and the vertical line represents the $2.5-97.5 \% \mathrm{Cl}$ of the score in that category. Within each factor, potential differences in mean score are tested using Student's $t$ test, setting the mean score in the first category as a reference $(r)$. We report the corresponding $p$ value above the boxplots for other groups. For readability, $p$ values were coded as * for $p$ values in $(0.05,0.01),{ }^{* *}$ for $p$ values in $(0.01,0.001)$ and ${ }^{* * *}$ for $p$ values.

consultation compared to phone consultations $(p<0.001)$ (Fig. 2). Multivariable analysis showed significant associations between TSQ score and anxiety level, as well as disease status and teleconsultation modality. Differences in mean TSQ was observed between "Possible anxiety", "anxiety" and "Severe anxiety" groups compared to "No/low anxiety" patients $(\beta=-3.8, p<0.001, \beta=$ $-2.8, p=0.022$ and $\beta=-6.4, p<0.001$, respectively) (Table 2 ). In terms of TSQ, the odds of not being satisfied among those with severe anxiety increased by a factor of 3.0 compared to those with no/low anxiety $(\mathrm{OR}=3.0,95 \% \mathrm{Cl} 1.7-5.0)$. The disease status was significantly associated with poor satisfaction, and those with standard follow-up were more satisfied than those with localised cancer (OR $=0.5,95 \% \mathrm{Cl} 0.3-0.7$ ) (Table 3 ).

\section{DISCUSSION}

As the first wave of the COVID-19 pandemic has disrupted healthcare systems across the world including France and Italy, many cancer centres have opted to limit hospital visits and meetings whenever possible $[20,21]$. Multidisciplinary meetings were maintained in a videoconference format to reduce the contact between healthcare professionals, which has been widely accepted among physicians involved in the management of breast cancer [22]. Patient visits were transformed to teleconsultations in many instances but the patient satisfaction with this consultation format was not reported [23]. This study provides a snapshot of the satisfaction of patients with breast cancer undergoing teleconsultation instead of in-person visits through an online questionnaire addressed to participants by email. In our experience, patients were predominantly aged $>50$ years $(72.6 \%)$, undergoing standard follow-up (53.3\%) and living in hotspot regions (69\%).

With the rapid spread of the COVID-19 pandemic, most inperson visits were switched to teleconsultations on short notice. Nevertheless, the anxiety scale system showed relatively low scores with almost half of the participants presenting no/low anxiety (HADS-A $\leq 7$ ). The EORTC OUT-PATSAT 35 and TSQ scores were considerably high and suggestive that the majority of the participants had a positive experience with teleconsultations. The lack of clinical breast exam is worrying for many patients, as outlined in the answers to the last two items of the questionnaire. Our findings adjusted for confounding factors showed that the two satisfaction scores correlated to the anxiety score. A meta- analysis of 2190 women with breast cancer showed a similar anxiety score between telehealth interventions and in-person visits; it associated telehealth intervention with a higher quality of life and self-efficacy as well as less depression and perceived stress [24]. The poor satisfaction EORTC OUT-PATSAT 35 score correlated to age and teleconsultation modality. Elderly patients are not commonly favourable to the use of technological tools to communicate medical results, which may impact the difference in video or phone preferences $[25,26]$. The poorer satisfaction of TSQ correlated mainly to disease status. The TSQ score was significantly lower among patients in follow-up for whom it is potentially more important to meet physically with their oncologists because of the less frequent consultations. The partially nonreproducible correlation findings between the two satisfaction scores may be explained by the fact that EORTC OUTPATSAT 35 mainly evaluates the global satisfaction with the health system, whereas TSQ is more specific and only related to telehealth itself. Surprisingly, the two satisfaction scores were not associated with living in COVID-19 hotspots or not, keeping in mind that the study was conducted before the widespread of COVID-19 vaccines.

In 2011, breast cancer survivors participating in a general survivorship survey considered that teleconsultation had a less favourable impact on cancer survival and cancer-related worrying compared to in-person visits [27]. On the other hand, several randomised trials have reported high satisfaction scores among patients with breast cancer undergoing nurse-led teleconsultations by phone and in-person visits before the COVID-19 pandemic [28-31]. Participants were satisfied with the interpersonal aspects, emotional functioning and feeling of control and anxiety [28-31]. The clinical implication of teleconsultations was also evaluated in a randomised trial of 374 women with breast cancer [30]. It showed that teleconsultations had higher levels of satisfaction compared to face-to-face consultations and were not shown to increase the anxiety level despite omitting clinical examinations. The time to detection of the few recurrences encountered (4.5\%) was not statistically different between traditional consults and teleconsultation by phone ( 60.5 vs 39.0 days; $p=0.228$ ). Notably, the number of clinical investigations did not differ between the two groups albeit the lack of visual cues [30]. Another clinical implication of teleconsultation may be the limited evidence supporting informed drug adherence in this setting, as reflected in the management of osteoporosis [32]. 
Table 2. Mean (and SE) contribution of different factors to the EORTC OUT-PATSAT 35 and TSQ scores (multivariable analysis).

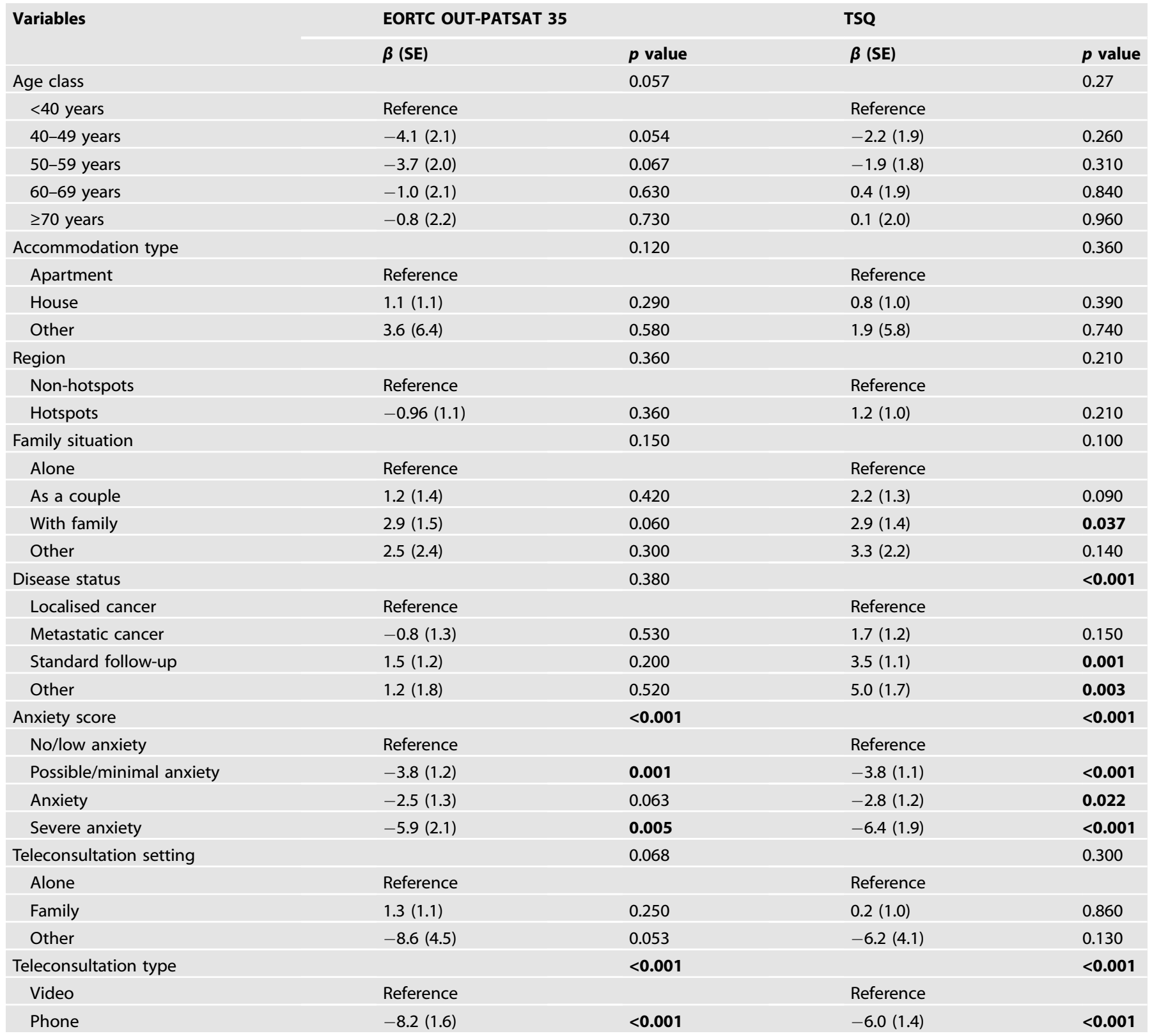

SE standard error, TSQ Telemedicine Satisfaction Questionnaire. Statistically significant findings are marked in bold.

There are several limitations inherent to the design of this study. First, the patient population may not represent the whole population of patients with breast cancer undergoing cancer care given that the majority consulted tertiary cancer centres. Second, many patients appreciated teleconsultation to avoid long travel distances and hospital exposure and transmission risk of COVID-19 $[33,34]$. However, the satisfaction with teleconsultation may be biased by several factors that were not directly measured. For instance, many elderly patients lacked any experience in using video-based teleconsultation, had hearing and cognitive difficulties and had inaccessibility to the results of biologic tests and imaging, which may result in negative feedback relative to teleconsultation $[35,36]$. In line with previously published data [37], patients who opted for video-based teleconsultations were younger, lived with their families and had localised disease
(Supplementary Table 1). Consultation reimbursements did not impact the implementation of either teleconsultation modalities, namely by phone or video-based call. Both phone- and videobased modalities were fully covered in France by the French Health Insurance organisation since the beginning of the pandemic [38], whereas neither modality was reimbursed in Italy at the time of the present survey [39]. In comparison to in-patient visit, published data showed that teleconsultations were shorter, had fewer instances of problems raised by patients and scored lower on consultation quality items [40]. Furthermore, our findings may be biased by a preponderance of patients who were likely experienced with the internet given that our invitations were addressed by email. Last, all items were self-collected, thus some items may not be completely accurate, in particular regarding disease stage assessment. 
Table 3. Association between different factors and poor satisfaction EORTC OUT-PATSAT 35 and TSQ scores (multivariable analysis).

\begin{tabular}{|c|c|c|c|c|}
\hline \multirow[t]{2}{*}{ Variables } & \multicolumn{2}{|c|}{ EORTC OUT-PATSAT 35} & \multicolumn{2}{|l|}{ TSQ } \\
\hline & OR $(95 \% \mathrm{Cl})$ & $p$ value & OR $(95 \% \mathrm{Cl})$ & $p$ value \\
\hline Age class & & 0.010 & & 0.630 \\
\hline$<40$ years & Reference & & Reference & \\
\hline $50-59$ years & $2.3(1.2-4.4)$ & 0.017 & $1.3(0.7-2.3)$ & 0.420 \\
\hline $60-69$ years & $1.4(0.7-2.8)$ & 0.360 & $1.0(0.5-1.9)$ & 0.960 \\
\hline$\geq 70$ years & $1.4(0.7-2.8)$ & 0.390 & $1.3(0.6-2.4)$ & 0.510 \\
\hline Apartment & Reference & & Reference & \\
\hline House & $0.8(0.6-1.1)$ & 0.250 & $0.8(0.6-1.1)$ & 0.150 \\
\hline Other & $1.0(0.2-5.6)$ & 0.990 & $0.4(0.05-3.9)$ & 0.460 \\
\hline Region & & 0.730 & & 0.550 \\
\hline Non-hotspots & Reference & & Reference & \\
\hline Hotspots & $1.1(0.8-1.4)$ & 0.730 & $0.9(0.7-1.2)$ & 0.550 \\
\hline Other & $0.7(0.4-1.4)$ & 0.290 & $1.2(0.6-2.4)$ & 0.590 \\
\hline Disease status & & 0.250 & & $<0.001$ \\
\hline Localised cancer & Reference & & Reference & \\
\hline Metastatic cancer & $0.9(0.6-1.4)$ & 0.730 & $0.8(0.6-1.2)$ & 0.300 \\
\hline Standard follow-up & $0.7(0.5-1.0)$ & 0.052 & $0.5(0.3-0.7)$ & $<0.001$ \\
\hline Other & $0.8(0.5-1.4)$ & 0.400 & $0.4(0.2-0.8)$ & 0.010 \\
\hline Anxiety score & & 0.039 & & $<0.001$ \\
\hline No/low anxiety & Reference & & Reference & \\
\hline Possible/minimal anxiety & $1.2(0.9-1.7)$ & 0.230 & $1.7(1.2-2.4)$ & 0.001 \\
\hline Anxiety & $1.2(0.8-1.8)$ & 0.270 & $1.5(1.0-2.2)$ & 0.033 \\
\hline Severe anxiety & $2.1(1.2-3.6)$ & 0.006 & $3.0(1.7-5.0)$ & $<0.001$ \\
\hline
\end{tabular}

$\mathrm{Cl}$ confidence interval, OR odds ratio, TSQ Telemedicine Satisfaction Questionnaire. Statistically significant findings are marked in bold.

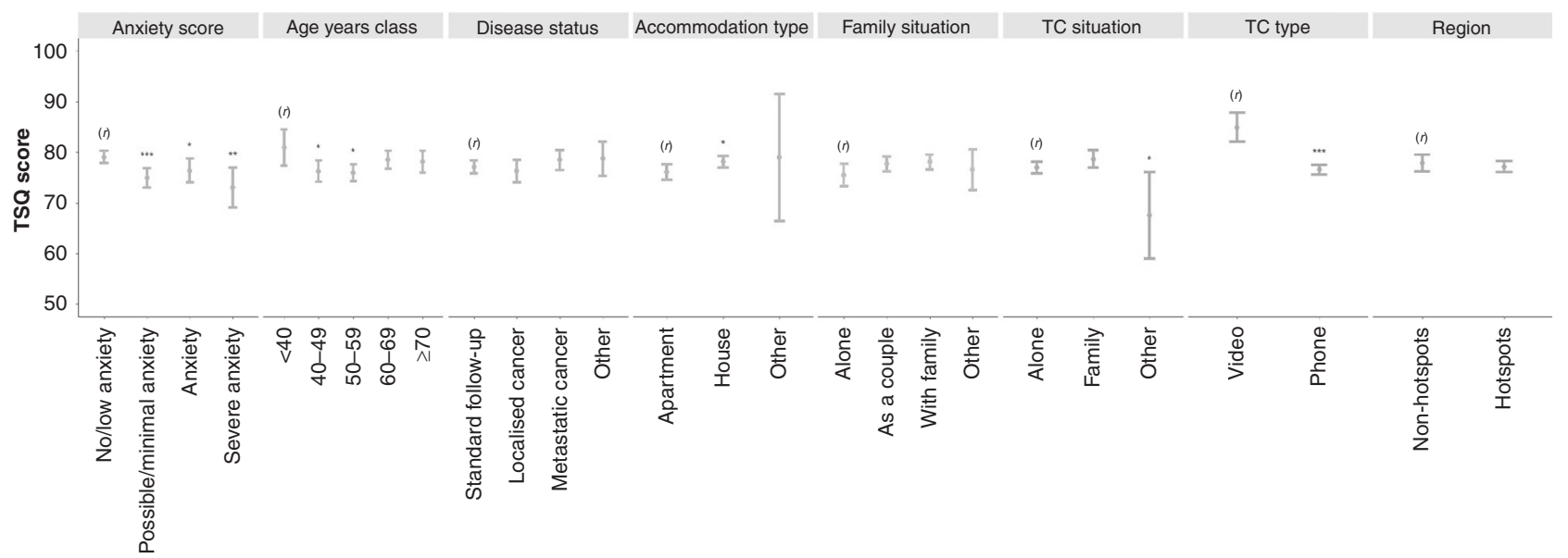

Fig. 2 Forest plot of the univariate analysis for the TSQ score distribution by different characteristic factors. For each factors' category, the point estimate of the mean score is represented by a bullet, and the vertical line represents the $2.5-97.5 \% \mathrm{Cl}$ of the score in that category. Within each factor, potential differences in mean score are tested using Student's $t$ test, setting the mean score in the first category as a reference $(r)$. We report the corresponding $p$ value above the boxplots for other groups. For readability, $p$ values were coded as * for $p$ values in $(0.05,0.01),{ }^{* *}$ for $p$ values in $(0.01,0.001)$ and ${ }^{* * *}$ for $p$ values. 
In conclusion, our findings suggest that patients with breast cancer were globally satisfied with teleconsultation during the first wave of the COVID-19 pandemic. With high satisfaction scores and a substantial reduction in hospital visits, teleconsultation may be an acceptable alternative follow-up modality in special circumstances. The economic evaluation of follow-up strategies after curative treatment for breast cancer considered that teleconsultations can be an appropriate cost-effective alternative to in-person visits after treatment completion [41]. Teleconsultations may reduce the burden on busy hospitals, especially during the pandemic, but do not necessarily lead to cost savings [42].

Although in-person visits remain the standard practice given the access to clinical examination, medical tests and imaging review and interpersonal relation between the oncologist and the patient, teleconsultations should be further evaluated to better identify the patients who are suitable for this strategy in special circumstances. Beyond organisation and satisfaction, high-level medical validation of their quality and adequacy is foundational before implementing structured teleconsultations in clinical practice. Our study is a first step that identifies populations more prone to accept and be satisfied with this approach. Further steps should describe the general mix of different consult modalities and compare the satisfaction score of inperson visits and teleconsultations by phone- or video-based calls.

\section{REFERENCES}

1. WHO. WHO coronavirus (COVID-19) dashboard. https://covid19.who.int. Accessed 24 Apr 2021.

2. Tangcharoensathien V, Bassett MT, Meng Q, Mills A. Are overwhelmed health systems an inevitable consequence of covid-19? Experiences from China, Thailand, and New York State. BMJ. 2021;372:n83. https://doi.org/10.1136/bmj.n83.

3. Patt D, Gordan L, Diaz M, Okon T, Grady L, Harmison M, et al. Impact of COVID-19 on cancer care: how the pandemic is delaying cancer diagnosis and treatment for American seniors. JCO Clin Cancer Informatics. 2020;1059-71. https://doi.org/ 10.1200/CCl.20.00134.

4. Horn L, Garassino M. COVID-19 in patients with cancer: managing a pandemic within a pandemic. Nat Rev Clin Oncol. 2021;18:1-2. https://doi.org/10.1038/ s41571-020-00441-5

5. Rassy E, Khoury-Abboud R-M, Ibrahim N, Kattan C, Assi T, Kattan J. What the oncologist needs to know about COVID-19 infection in cancer patients. Future Oncol. 2020;16:1153-6. https://doi.org/10.2217/fon-2020-0312

6. Al-Shamsi HO, Alhazzani W, Alhuraiji A, Coomes EA, Chemaly RF, Almuhanna M, et al. A practical approach to the management of cancer patients during the novel coronavirus disease 2019 (COVID-19) pandemic: An International Collaborative Group. Oncologist. 2020;25:e936-45. https://doi.org/10.1634/ theoncologist.2020-0213

7. Curigliano G, Banerjee S, Cervantes A, Garassino MC, Garrido P, Girard N, et al. Managing cancer patients during the COVID-19 pandemic: an ESMO multidisciplinary expert consensus. Ann Oncol. 2020;31:1320-35. https://doi.org/ 10.1016/j.annonc.2020.07.010

8. Jazieh AR, Chan SL, Curigliano G, Dickson N, Eaton V, Garcia-Foncillas J, et al. Delivering cancer care during the COVID-19 pandemic: recommendations and lessons learned from ASCO global webinars. JCO Glob Oncol. 2020;6:1461-71. https://doi.org/10.1200/GO.20.00423

9. van de Haar J, Hoes LR, Coles CE, Seamon K, Fröhling S, Jäger D, et al. Caring for patients with cancer in the COVID-19 era. Nat Med. 2020;26:665-71. https://doi. org/10.1038/s41591-020-0874-8

10. Poggio F, Tagliamento M, Di Maio M, Martelli V, De Maria A, Barisione E, et al. Assessing the impact of the COVID-19 outbreak on the attitudes and practice of italian oncologists toward breast cancer care and related research activities. JCO Oncol Pract. 2020;16:e1304-14. https://doi.org/10.1200/OP.20.00297

11. Liu R, Sundaresan T, Reed ME, Trosman JR, Weldon CB, Kolevska T. Telehealth in oncology during the COVID-19 outbreak: bringing the house call back virtually. JCO Oncol Pract. 2020;16:289-93. https://doi.org/10.1200/OP.20.00199

12. ESMO. ESMO management and treatment adapted recommendations in the COVID-19 era: breast cancer. https://www.esmo.org/guidelines/cancer-patientmanagement-during-the-covid-19-pandemic/breast-cancer-in-the-covid-19-era. Accessed 24 Apr 2021.

13. Gligorov J, Bachelot T, Pierga J-Y, Antoine E-C, Balleyguier C, Barranger E. et al. [COVID-19 and people followed for breast cancer: French guidelines for clinical practice of Nice-St Paul de Vence, in collaboration with the Collège Nationale des
Gynécologues et Obstétriciens Français (CNGOF), the Société d'Imagerie de la Femme (SIFEM), the Société Française de Chirurgie Oncologique (SFCO), the Société Française de Sénologie et Pathologie Mammaire (SFSPM) and the French Breast Cancer Intergroup-UNICANCER (UCBG)]. Bull Cancer. 2020;107:528-37. https://doi.org/10.1016/j.bulcan.2020.03.008.

14. Tagliamento M, Agostinetto E, Bruzzone M, Ceppi M, Saini KS, de Azambuja E, et al. Mortality in adult patients with solid or hematological malignancies and SARS-CoV-2 infection with a specific focus on lung and breast malignancies: a systematic review and meta-analysis. Crit Rev Oncol Hematol. 2021;103365, https://doi.org/10.1016/j.critrevonc.2021.103365.

15. Poinsot R, Altmeyer A, Conroy T, Savignoni A, Asselain B, Léonard I. et al. [Multisite validation study of questionnaire assessing out-patient satisfaction with care questionnaire in ambulatory chemotherapy or radiotherapy treatment]. Bull Cancer. 2006;93:315-27.

16. Pinar U, Anract J, Perrot O, Tabourin T, Chartier-Kastler E, Parra J et al. Preliminary assessment of patient and physician satisfaction with the use of teleconsultation in urology during the COVID-19 pandemic. World J Urol. 2020. https://doi.org/ 10.1007/s00345-020-03432-4.

17. Yip MP, Chang AM, Chan J, MacKenzie AE. Development of the Telemedicine Satisfaction Questionnaire to evaluate patient satisfaction with telemedicine: a preliminary study. J Telemed Telecare. 2003;9:46-50. https://doi.org/10.1258/ 135763303321159693

18. Bjelland I, Dahl AA, Haug TT, Neckelmann D. The validity of the Hospital Anxiety and Depression Scale. Update Lit Rev J Psychosom Res. 2002;52:69-77. https:// doi.org/10.1016/s0022-3999(01)00296-3

19. Bocéréan C, Dupret E. A validation study of the Hospital Anxiety and Depression Scale (HADS) in a large sample of French employees. BMC Psychiatry. 2014;14:354 https://doi.org/10.1186/s12888-014-0354-0

20. Demartini B, Nisticò V, D’Agostino A, Priori A, Gambini O. Early psychiatric impact of COVID-19 pandemic on the general population and healthcare workers in Italy: a preliminary study. Front Psychiatry. 2020;11:561345. https://doi.org/10.3389/ fpsyt.2020.561345.

21. Annane D, Federici L, Chagnon J-L, Diehl JL, Dreyfuss D, Guiot $P$, et al. Intensive care units, the Achilles heel of France in the COVID-19 battle. Lancet Reg Health Eur. 2021;2:100046. https://doi.org/10.1016/j.lanepe.2021.100046.

22. Cathcart P, Smith S, Clayton G. Strengths and limitations of video-conference multidisciplinary management of breast disease during the COVID-19 pandemic. Br J Surg. 2020. https://doi.org/10.1093/bjs/znaa046

23. Paterson C, Bacon R, Dwyer R, Morrison KS, Toohey K, O'Dea A, et al. The role of telehealth during the COVID-19 pandemic across the interdisciplinary cancer team: implications for practice. Semin Oncol Nurs. 2020;36:151090. https://doi. org/10.1016/j.soncn.2020.151090

24. Chen Y-Y, Guan B-S, Li Z-K, Li X-Y. Effect of telehealth intervention on breast cancer patients' quality of life and psychological outcomes: a meta-analysis. J Telemed Telecare. 2018;24:157-67. https://doi.org/10.1177/1357633X16686777

25. Onuma $A E$, Palmer Kelly $E$, Chakedis J, Paredes AZ, Tsilimigras DI, Wiemann B, et al. Patient preferences on the use of technology in cancer surveillance after curative surgery: a cross-sectional analysis. Surgery. 2019;165:782-8. https://doi. org/10.1016/j.surg.2018.12.021

26. van den Berg N, Schumann M, Kraft K, Hoffmann W. Telemedicine and telecare for older patients-a systematic review. Maturitas. 2012;73:94-114. https://doi. org/10.1016/j.maturitas.2012.06.010

27. Mayer EL, Gropper AB, Neville BA, Partridge AH, Cameron DB, Winer EP et al. Breast cancer survivors' perceptions of survivorship care options. J Clin Oncol. 2011. https://doi.org/10.1200/JCO.2011.36.9264.

28. Kimman ML, Bloebaum MM, Dirksen CD, Houben RM, Lambin P, Boersma LJ. Patient satisfaction with nurse-led telephone follow-up after curative treatment for breast cancer. BMC Cancer. 2010;10:174 https://doi.org/10.1186/1471-240710-174

29. Kimman ML, Dirksen CD, Voogd AC, Falger P, Gijsen BCM, Thuring M, et al. Nurseled telephone follow-up and an educational group programme after breast cancer treatment: results of a $2 \times 2$ randomised controlled trial. Eur J Cancer. 2011;47:1027-36. https://doi.org/10.1016/j.ejca.2010.12.003

30. Beaver K, Tysver-Robinson D, Campbell M, Twomey M, Williamson S, Hindley A, et al. Comparing hospital and telephone follow-up after treatment for breast cancer: randomised equivalence trial. BMJ. 2009;338:a3147 https://doi.org/ 10.1136/bmj.a3147

31. Beaver K, Williamson S, Chalmers K. Telephone follow-up after treatment for breast cancer: views and experiences of patients and specialist breast care nurses. J Clin Nurs. 2010;19:2916-24. https://doi.org/10.1111/j.13652702.2010.03197.x

32. Paskins Z, Crawford-Manning F, Bullock L, Jinks C. Identifying and managing osteoporosis before and after COVID-19: rise of the remote consultation? Osteoporos Int. 2020;31:1629-32. https://doi.org/10.1007/s00198-020-05465-2 
33. Richterman A, Meyerowitz EA, Cevik M. Hospital-acquired SARS-CoV-2 infection: lessons for public health. JAMA. 2020;324:2155-6. https://doi.org/10.1001/ jama.2020.21399

34. Rickman HM, Rampling $T$, Shaw $K$, Martinez-Garcia G, Hail L, Coen $P$, et al. Nosocomial transmission of coronavirus disease 2019: a retrospective study of 66 hospital-acquired cases in a London teaching hospital. Clin Infect Dis. 2021;72:690-3. https://doi.org/10.1093/cid/ciaa816

35. Narayanan S, Lopez G, Liu W, Cohen A, Cohen L. The use of mobile-technologies to deliver integrative medicine during and beyond the COVID-19 world pandemic. Glob Adv Health Med. 2020;9:2164956120977437. https://doi.org/10.1177/ 2164956120977437.

36. Narayanan S, Lopez G, Powers-James C, Fellman BM, Chunduru A, Li Y, et al. Integrative oncology consultations delivered via telehealth in 2020 and in-person in 2019: paradigm shift during the COVID-19 world pandemic. Integr Cancer Ther 2021:20:1534735421999101. https://doi.org/10.1177/1534735421999101.

37. Neeman E, Lyon L, Sun H, Conell CA, Reed M, Kumar D, et al. The future of teleoncology: trends and disparities in telehealth and secure message utilization in the COVID-19 era. J Clin Oncol. 2021;39:1506. https://doi.org/10.1200/ JCO.2021.39.15_suppl.1506

38. Haute Autorité de Santé. 2021 https://www.has-sante.fr/upload/docs/application/ pdf/2020-04/reponses_rapides_dans_le_cadre_du_covid-19_teleconsultation_ et_telesoin.pdf. Accessed 3 Aug 2021.

39. Omboni S. Telemedicine during the COVID-19 in Italy: a missed opportunity? Telemed e-Health. 2020;26:973-5. https://doi.org/10.1089/tmj.2020.0106

40. Hammersley V, Donaghy E, Parker R, McNeilly $H$, Atherton $H$, Bikker A, et al. Comparing the content and quality of video, telephone, and face-to-face consultations: a non-randomised, quasi-experimental, exploratory study in UK primary care. Br J Gen Pract. 2019;69:e595-604. https://doi.org/10.3399/ bjgp19X704573

41. Kimman $M L$, Dirksen $C D$, Voogd $A C$, Falger $P$, Gijsen $B C M$, Thuring $M$, et al Economic evaluation of four follow-up strategies after curative treatment for breast cancer: results of an RCT. Eur J Cancer. 2011;47:1175-85. https://doi.org/ 10.1016/j.ejca.2010.12.017

42. Beaver K, Hollingworth W, McDonald R, Dunn G, Tysver-Robinson D, Thomson L, et al. Economic evaluation of a randomized clinical trial of hospital versus telephone follow-up after treatment for breast cancer. Br J Surg. 2009;96:1406-15. https://doi.org/10.1002/bjs.6753

\section{ACKNOWLEDGEMENTS}

We deeply thank all the participants who kindly accepted to fill this survey, the Patient Committee at Gustave Roussy who advised us on the survey and the medical assistants who helped sending the emails.

\section{AUTHOR CONTRIBUTIONS}

$A B, S D, B P, E R$ : concept of the study and review of the literature. MK: statistical analysis. $E R, M K, B P$ : writing of the initial draft. $P E H, C L, J-Y P, S D, M L, B P$ : critical revision of the manuscript. All authors: collection of data and approval of the final version of this manuscript.

\section{ETHICS APPROVAL AND CONSENT TO PARTICIPATE}

The online questionnaire was completely anonymous. An information letter notified the participants that completing the questionnaire infers an inherent agreement to their participation in the study and subsequently a written consent was not required. The study was examined and approved by the internal review board (IRB) at Gustave Roussy, Villejuif, France on April 6, 2020. The study was performed in accordance with the Declaration of Helsinki.

\section{COMPETING INTERESTS}

$A B, M K, E R, C L$, LV, CU, ED, DG, MS, SG, JG, AP, VD, J-YP, TP, SL, WJ, M-AB, ADJ, SD: None relevant to this study. $P E H$ reports grants, personal fees and non-financial support from PFIZER, grants and non-financial support from NOVARTIS, grants and non-financial support from ROCHE, grants, personal fees and non-financial support from AstraZeneca, personal fees and non-financial support from Mylan, grants, personal fees and non-financial support from Pierre Fabre, personal fees and nonfinancial support from AMGEN and personal fees and non-financial support from SEAGEN. ML acted as a consultant for Roche, Lilly, Novartis and AstraZeneca and received honoraria from Sandoz, Roche, Lilly, Pfizer and Novartis outside the submitted work. BP: consulting/advisor: Puma Biotechnology, Novartis, Myriad Genetics, Pierre Fabre; personal fees: Novartis, AstraZeneca, MSD Oncology, Pfizer research funding: Daiichi-Sankyo, Puma Biotechnology, Novartis, Merus, Pfizer, AstraZeneca.

\section{ADDITIONAL INFORMATION}

Supplementary information The online version contains supplementary material available at https://doi.org/10.1038/s41416-021-01555-y.

Correspondence and requests for materials should be addressed to Barbara Pistilli.

Reprints and permission information is available at http://www.nature.com/ reprints

Publisher's note Springer Nature remains neutral with regard to jurisdictional claims in published maps and institutional affiliations. 